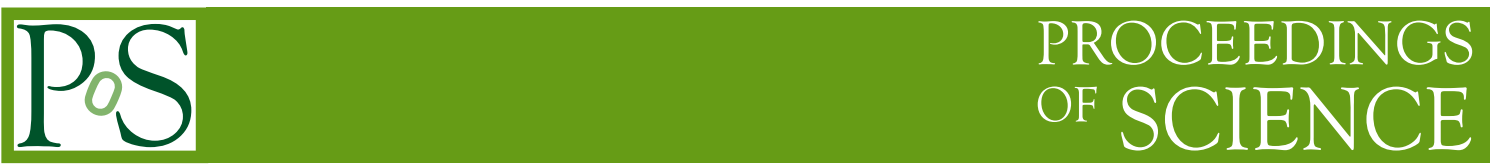

\title{
One flavor mass reweighting: foundations
}

\author{
Björn Leder ${ }^{* a, b}$, Jacob Finkenrath ${ }^{a}$ and Francesco Knechtli ${ }^{a}$ \\ ${ }^{a}$ Department of Physics, Bergische Universität Wuppertal \\ Gaussstr. 20, D-42119 Wuppertal, Germany \\ ${ }^{b}$ Department of Mathematics, Bergische Universität Wuppertal \\ Gaussstr. 20, D-42119 Wuppertal, Germany \\ E-mail: leder@physik.uni-wuppertal.de
}

\begin{abstract}
Reweighting is not a new method in lattice QCD, but a comprehensive analysis is missing in the literature. We close this gap by presenting: (i) a proof of an integral representation of the complex determinant of a complex matrix, (ii) a method to control the stochastic error of its Monte Carlo estimation, (iii) expansions of the stochastic error and the ensemble fluctuations of the one flavor reweighting factor. Based on (iii) we present a detailed scaling analysis and optimized reweighting strategies. As an application we analyze the ensemble fluctuations of the reweighting factor corresponding to the sea contribution to isospin splitting and predict at physical quark masses a standard deviation of $\pm 20 \%$.
\end{abstract}

31st International Symposium on Lattice Field Theory - LATTICE 2013

July 29 - August 3, 2013

Mainz, Germany

${ }^{*}$ Speaker. 


\section{Introduction}

In lattice QCD observables are computed as expectation values $\langle\mathscr{O}\rangle$ of composite fields $\mathscr{O}$. The brackets stand for the average over an ensemble of gauge configurations that is generated according to a probability distribution $P$. Since $P$ depends on all the bare parameters of QCD a new ensemble has to be generated for each set of bare parameters, e.g., to study the continuum limit or the mass dependence of observables. However, it is also possible to obtain $\langle\mathscr{O}\rangle_{P^{\prime}}$ if $P^{\prime}=P \cdot W$, because then

$$
\langle\mathscr{O}\rangle_{P^{\prime}}=\langle\mathscr{O} W\rangle /\langle W\rangle
$$

The variance of the reweighting factor $W$ limits the applicability of reweighting. If its fluctuations become too large a reliable error estimation is impossible: only a few configurations out of a finite ensemble of configurations from a Monte-Carlo simulation will dominate the mean, i.e., the socalled overlap problem emerges.

Assuming $W=1 / \operatorname{det}\left(I+\varepsilon D^{-1} X\right)$ with some complex matrices $D, X$ and real scalar $\varepsilon$, which is the case for mass reweighting, we can expand the variance $\operatorname{var}(x)=\left\langle x^{2}\right\rangle-\langle x\rangle^{2}$

$$
\sigma_{W}^{2} \equiv \operatorname{var}(W) /\langle W\rangle^{2}=\varepsilon^{2} \operatorname{var}\left(\operatorname{Tr}\left(D^{-1} X\right)\right)+\mathrm{O}\left(\varepsilon^{3}\right) .
$$

We will come back to this expression in Section 4. But first we need to specify how to numerically evaluate the reweighting factor $W$, i.e., the determinant of a complex matrix. Direct computation is impossible since $D$ will turn out to be the lattice Dirac operator. Instead we write in Section 2 the determinant as an integral and briefly describe a proof for its existence for general complex matrices $A$ if $A+A^{\dagger}$ is positive definite. With this proof at hand we define an unbiased and robust stochastic estimation of the integral in Section 3. The application of these results to mass reweighting and numerical results for the scaling of the fluctuations are presented in Sections 4-6.

\section{Integral representation of the determinant}

Let $A \in \mathbb{C}^{n \times n}$ and $\eta \in \mathbb{C}^{n}$ and $\sigma(A)$ the eigenvalues of $A$. Then one can show

$$
\frac{1}{\operatorname{det} A}=\int \mathrm{D}[\eta] \mathrm{e}^{-\eta^{\dagger} A \eta} \quad \text { iff } \quad \lambda>0, \forall \lambda \in \sigma\left(A+A^{\dagger}\right)
$$

with $\eta=\eta_{1} e_{1}+\cdots+\eta_{n} e_{n}, \eta_{i} \in \mathbb{C},\left\{e_{1}, \ldots, e_{n}\right\}$ an orthonormal basis of $\mathbb{C}^{n}$ and

$$
\mathrm{D}[\eta]=\prod_{i=1}^{n} \frac{\mathrm{dRe}\left(\eta_{i}\right) \mathrm{d} \operatorname{Im}\left(\eta_{i}\right)}{\pi} .
$$

For normal matrices the proof is straightforward since then $A$ is diagonalizable by a unitary matrix, i.e., $A=U^{\dagger} \Lambda U$ with $U^{\dagger} U=I$ and $\Lambda=\operatorname{diag}\left(\lambda_{1}, \ldots, \lambda_{n}\right)$. The transformation $\eta \rightarrow U^{\dagger} \eta$ has a Jacobian determinant of one and simplifies the multi-dimensional integral to a product of two-dimensional integrals

$$
\int \mathrm{D}[\eta] \mathrm{e}^{-\eta^{\dagger} A \eta}=\prod_{i=1}^{n} \int \frac{\mathrm{d} x_{i} \mathrm{~d} y_{i}}{\pi} \mathrm{e}^{-\lambda_{i}\left(x_{i}^{2}+y_{i}^{2}\right)} \quad \text { iff } A \text { normal } .
$$

The latter is defined iff $\operatorname{Re}\left(\lambda_{i}\right)>0$ and can be solved via polar coordinates giving $1 / \lambda_{i}$. Since $A=U^{\dagger} \Lambda U$ we have $\sigma\left(A+A^{\dagger}\right)=\sigma(2 \operatorname{Re}(\Lambda))$, thus proving Eq. (2.1). 
For non-normal $A$, e.g. the Wilson Dirac operator or functions thereof, there is no unitary transformation that diagonalizes $A$. In [1] a proof was given based on the Schur decomposition of $A=Q^{\dagger}(\Lambda+K) Q$, with unitary $Q$ and strictly upper tridiagonal $K$. Surely we have $\operatorname{det}(A)=$ $\operatorname{det}(\Lambda+K)=\operatorname{det}(\Lambda)=\operatorname{det}\left(U^{\dagger} \Lambda U\right)$ for all unitary $U$, i.e., the determinant of a non-normal matrix $A$ is equal to the determinant of a normal matrix with the same eigenvalues. The proof shows that if the integrand is absolute convergent, i.e., if $\lambda \in \sigma\left(A+A^{\dagger}\right)>0$, the integral representation of $1 / \operatorname{det}(\Lambda+K)$ is equal to that of $1 / \operatorname{det}(\Lambda)$ by variable substitution.

\section{Stochastic estimator}

If the integral representation of the determinant Eq. (2.1) exists an unbiased stochastic estimator of $W(A)=1 / \operatorname{det}(A)$ is given by

$$
W_{N_{\eta}}(A)=\frac{1}{N_{\eta}} \sum_{k=1}^{N_{\eta}} e^{-\eta^{(k)^{\dagger}}(A-I) \eta^{(k)}},
$$

with Gaussian distributed ${ }^{1}$ random vectors $\left\{\eta^{(1)}, \ldots, \eta^{\left(N_{\eta}\right)}\right\}$. By the rules of Monte-Carlo integration $W_{N_{\eta}}$ differs from $W$ by terms of $\mathrm{O}\left(1 / \sqrt{N_{\eta}}\right)$. Since this estimator can be complex we define the variance as $\sigma_{\eta}^{2}(A)=\left\langle\left\langle W_{N_{\eta}}(A) W_{N_{\eta}}(A)^{*}\right\rangle\right\rangle-\left\langle\left\langle W_{N_{\eta}}(A)\right\rangle\right\rangle\left\langle\left\langle W_{N_{\eta}}(A)\right\rangle\right\rangle^{*}$, where $\langle\langle O\rangle\rangle=$ $\int \mathrm{D}[\eta] \exp \left(-\eta^{\dagger} \eta\right) O$. It is explicitly given by the integral representation of

$$
\sigma_{\eta}^{2}(A)=\frac{1}{\operatorname{det}\left(A+A^{\dagger}-1\right)}-\frac{1}{\operatorname{det}\left(A A^{\dagger}\right)} .
$$

Therefore the variance exists iff $\sigma\left(A+A^{\dagger}\right)>1$ (see [1] for a proof). If the matrix $A$ can be written as $A=I+\varepsilon B$ with $\varepsilon>0$ and $\varepsilon|| B \| \ll 1$ the relative error $\delta_{\eta}^{2}=\sigma_{\eta}^{2} /\left(N_{\eta}|W|^{2}\right)$ can be expanded as

$$
\delta_{\eta}^{2}(A)=\frac{1}{N_{\eta}}\left[\varepsilon^{2} \operatorname{Tr}\left(B B^{\dagger}\right)+\mathrm{O}\left(\varepsilon^{3}\right)\right] .
$$

For the validity of the estimator in Eq. (3.1) it is enough to ensure $\sigma\left(A+A^{\dagger}\right)>0$, since then formally $W_{N_{\eta}} \rightarrow W$ for $N_{\eta} \rightarrow \infty$. However, in numerical evaluations where only a small finite number of random vectors is affordable, a well defined and controlled error with an expansion as in (3.3) is indispensable. An unbiased stochastic estimator that fulfills these conditions automatically can be based on a factorization of $A$ (and thus $1 / \operatorname{det}(A)$ ). Assume $A=I+\varepsilon D^{-1} X$ with $\varepsilon\left\|D^{-1} X\right\| \gtrsim$ 1. Then for $N \geq 1$

$$
A=\prod_{i=0}^{N-1}\left[I+\left(\delta_{i+1}-\delta_{i}\right) D_{i}^{-1} X\right] \quad \text { with } \quad D_{i}=D+\delta_{i} X, \quad \delta_{0}=0, \quad \delta_{N}=\varepsilon,
$$

if $D_{i}$ is invertible for all $i=0, \ldots, N-1$. Note that $A$ can be written as a ratio $A=D_{N} / D_{0}$ and Eq. (3.4) as a product of ratios $A=\prod_{i} D_{i+1} / D_{i}$. The determinant $W(A)$ factorizes in the same way and the unbiased estimator is given by

$$
W_{N_{\eta}, N}(A)=\prod_{i=0}^{N-1} W_{N_{\eta}}\left(I+\bar{\varepsilon}_{i} D_{i}^{-1} X\right), \quad \bar{\varepsilon}_{i}=\delta_{i+1}-\delta_{i}
$$

\footnotetext{
${ }^{1}$ Other choices are possible. See [1] for a more general expression.
} 
where each factor uses an independent set of random vectors. In the view of the boundary conditions in Eq. (3.4) and the aim of minimizing the stochastic error in Eq. (3.3), the sequence $D_{0}, \ldots, D_{N-1}$ should be taken as discrete steps along a smooth interpolation between $D_{0}$ and $D_{N-1}$ with $\bar{\varepsilon}_{i} \rightarrow 0$ for $N \rightarrow \infty$. In the simplest case, which we assume here, it is a linear interpolation and $\bar{\varepsilon}_{i} \equiv \varepsilon / N$. Since the eigenvalues of $I+\bar{\varepsilon}_{i} D_{i}^{-1} X$ lie in the complex plane within a circle around one whose radius is shrinking to zero as $N \rightarrow \infty$, the condition for the variance Eq. (3.2) to exist and the expansion in Eq. (3.3) to converge for each factor in Eq. (3.5) can always be fulfilled by choosing a large enough $N$. Because each factor is an independent estimator, the overall relative error is given by simple error propagation and we obtain

$$
\delta_{\eta}^{2}(A)=\frac{1}{N_{\eta}} \sum_{i=0}^{N-1}\left[\bar{\varepsilon}_{i}^{2} \operatorname{Tr}\left(\left(D_{i} D_{i}^{\dagger}\right)^{-1} X X^{\dagger}\right)+\mathrm{O}\left(\bar{\varepsilon}_{i}^{3}\right)\right] \stackrel{N \rightarrow \infty}{\leq} \frac{\varepsilon^{2}}{N N_{\eta}} \max _{i} \operatorname{Tr}\left(\left(D_{i} D_{i}^{\dagger}\right)^{-1} X X^{\dagger}\right) .
$$

Thus for large enough $N$ the remaining condition for the existence of the estimator and the variance is that $D_{i}$ is invertible for all $i=0, \ldots, N-1$.

\section{Mass reweighting in lattice QCD}

We consider mass reweighting in lattice QCD with the lattice Dirac operator $D_{m}=D_{0}+m$, where $D_{0}$ is the operator at zero bare mass. Although mainly independent of the specific fermion discretization we have $\mathrm{O}(a)$-improved Wilson fermions in mind, for which we will present some results in Sections 5 and 6.

\subsection{One and two flavor reweighting}

The reweighting factors are determinants of ratios of Dirac operators at different mass parameters $m$. We here consider one and two flavor reweighting, which covers a wide range of applications of mass reweighting in lattice QCD. The matrix $A=I+\varepsilon D^{-1} X$ in the general formulas above is given by

$$
A_{1 \mathrm{f}}=D_{m_{s}-\Delta m}^{-1} D_{m_{s}}=I+\Delta m D_{m_{s}-\Delta m}^{-1},
$$

for one flavor reweighting and

$$
A_{2 \mathrm{f}}=\left(D_{m_{r}-\gamma \Delta m} D_{m_{s}+\Delta m}\right)^{-1} D_{m_{r}} D_{m_{s}}=I+\Delta m\left(D_{m_{s}+\Delta m} D_{m_{r}-\gamma \Delta m}\right)^{-1}\left(\gamma \Delta m+\gamma D_{m_{s}}-D_{m_{r}}\right),
$$

for two flavor reweighting. In each case $m_{r, s}$ are the ensemble mass parameters and $m_{s} \pm \Delta m$ and $m_{r}-\gamma \Delta m$, respectively, are the target mass parameters. For a detailed discussion of Eq. (4.2) we refer to [1]. The special case $m_{r}=m_{s}$ and $\gamma=-1$, i.e., reweighting of a mass-degenerated pair of quarks, was considered in [2]. Here we concentrate on the special case $m_{r}=m_{s}$ and $\gamma=1$, which we dub isospin reweighting

$$
A_{ \pm}=I+\Delta m^{2}\left(D_{m_{s}}^{2}-\Delta m^{2}\right)^{-1} .
$$

\subsection{Twisted mass reweighting}

Applying the estimator of Section 3 we have to ensure that $D_{m}$ is invertible for all occurring values of $m$ and for all configurations of a given ensemble. For Wilson fermions this can in general not be guaranteed. A solution is to add a small twisted mass $D_{0} \rightarrow D_{0}+i \gamma_{5} \mu$, because then the lattice Dirac operator has a gap. If a finite twisted mass is already included in the simulation as proposed in [3], this does not mean any additional effort. If the ensemble was generated at $\mu=0$ an additional reweighting to finite $\mu$ and back is necessary. In [1] we proposed to do this only for those configurations that suffered from small eigenvalues of $D_{m}$ for $\mu=0$. However, to ensure the 


\begin{tabular}{ccccc}
\hline & $V / a^{4}$ & $m_{\mathrm{PS}}$ in $\mathrm{MeV}$ & $\bar{m}$ in $\mathrm{MeV}$ & $\max (|\Delta \bar{m}|)$ in $\mathrm{MeV}$ \\
\hline D5 & $48 \times 24^{3}$ & 440 & 32 & 3 \\
F7 & $96 \times 48^{3}$ & 270 & 12 & 3 \\
\hline
\end{tabular}

Table 1: Ensembles and maximal reweighting distance considered for the numerical results. The pion masses $m_{\mathrm{PS}}$ and the renormalized quark masses $\bar{m}$ are rounded to steps of $5 \mathrm{MeV}$ and $1 \mathrm{MeV}$, respectively.

interchangeability of the integral over gauge fields and random vectors, it is necessary to do this for every configuration ${ }^{2}$. The analysis of the fluctuations and stochastic estimators in Section 3 can be applied to the case of twisted mass reweighting as well and is currently under investigation.

\subsection{Fluctuations and stochastic error}

Assuming $\Delta m>0$ and $m=m_{s}-\Delta m$ we obtain for the relative stochastic error and the fluctuations

$$
\left\langle\delta_{p}^{2}\right\rangle \approx \frac{\Delta m^{2 p}}{N N_{\eta}}\left\langle\operatorname{Tr}\left(\left(D_{m} D_{m}^{\dagger}\right)^{-p}\right)\right\rangle \quad \text { and } \quad \sigma_{p}^{2} \approx \Delta m^{2 p} \operatorname{var}\left(\operatorname{Tr}\left(D_{m}^{-p}\right)\right) .
$$

with $p=1$ for one flavor and $p=2$ for isospin reweighting. Some insight into the volume and (renormalized) quark mass dependence of the involved traces can in principal be obtained from chiral perturbation theory, e.g., as in [4]. For example, at lowest order in the chiral expansion

$$
\left\langle\operatorname{Tr}\left(\left(D_{m} D_{m}^{\dagger}\right)^{-p}\right)\right\rangle=\frac{\bar{m} \Sigma V}{\bar{m}^{2 p}} \frac{\Gamma\left(p-\frac{1}{2}\right)}{\sqrt{\pi} \Gamma(p)},
$$

with the volume $V$, the chiral condensate $\Sigma$ and the renormalized quark mass $\bar{m}$. In lack of an explicit calculation for $\operatorname{var}\left(\operatorname{Tr}\left(D_{m}^{-p}\right)\right)$ we assume the ad-hoc scaling formula $k \cdot\left(V / a^{4}\right) /\left(a^{q-2 p} \bar{m}^{q}\right)$ with some dimensionless constant $k$ and some power $q$ of the renormalized quark mass.

\section{One flavor reweighting}

In [1] numerical results where presented for mass reweighting of one ensemble, tagged D5, of $N_{\mathrm{f}}=2 \mathrm{O}(a)$-improved Wilson fermions at a lattice spacing of $a=0.066 \mathrm{fm}$ [5]. Here we add a second ensemble, tagged F7, at a smaller pion mass $m_{\mathrm{PS}}$ and with lager volume to the analysis. The ensemble was generated within the CLS effort ${ }^{3}$. We list the lattice volumes, the pion masses and the renormalized quark masses, as defined in [5], for the two ensembles in Tab. 1. We also give the maximal renormalized reweighting distance, where $\Delta \bar{m}$ is the difference of the renormalized quark masses before and after reweighting $\Delta \bar{m}=\bar{m}(m)-\bar{m}(m-\Delta m)$.

For the reweighting distances considered here the expansions in Eqs. (1.2) and (3.6) work and are dominated by the first term. We demonstrate this in the left panel of Fig. 1 for the ensemble fluctuations. The behavior of the stochastic error is similar. The two ensembles at different quark masses allow for a study of the quark mass dependence of the reweighting factor. As explained in Section 4 we expect for one flavor reweighting

$$
\left\langle\delta_{1 \mathrm{f}}^{2}\right\rangle \approx \frac{k_{\eta, 1 \mathrm{f}}}{N N_{\eta}} \frac{\Delta \bar{m}^{2} V}{a^{(2+q)} \bar{m}^{q}} \quad \text { and } \quad \sigma_{1 \mathrm{f}}^{2} \approx k_{1 \mathrm{f}} \frac{\Delta \bar{m}^{2} V}{a^{\left(2+q^{\prime}\right)} \bar{m}^{q^{\prime}}},
$$

\footnotetext{
${ }^{2}$ We thank M. Lüscher and S. Schaefer for pointing this out.

${ }^{3}$ https://twiki.cern.ch/twiki/bin/view/CLS/
} 

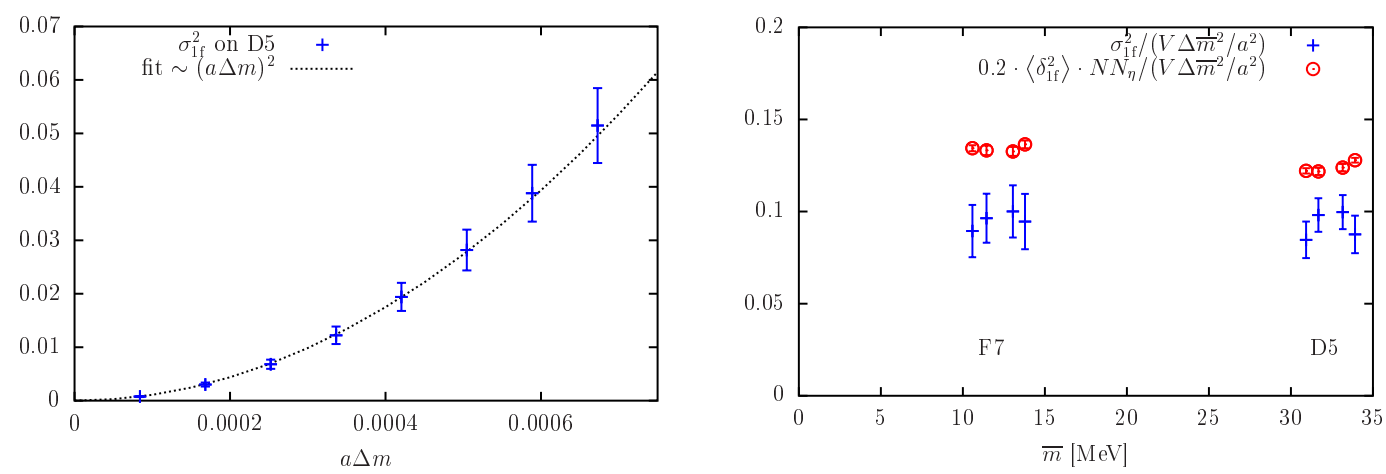

Figure 1: Left: Dependence of the ensemble fluctuations $\sigma_{1 \mathrm{f}}^{2}$ of one flavor reweighting on the reweighting distance $\Delta m$ in lattice units for ensemble D5. Right: Dependence of $\sigma_{1 \mathrm{f}}^{2}$ and the stochastic error $\delta_{1 \mathrm{f}}^{2}$ on the renormalized quark mass $\bar{m}$. Both quantities are normalized such that differences in the volume and the renormalized reweighting distance $\Delta \bar{m}$ are removed. The stochastic error $\left\langle\delta_{1 \mathrm{f}}^{2}\right\rangle$ is multiplied by 0.2 for visibility. Out of the 8 points in the left panel we plot the one with the largest $a \Delta m$, the one at half this distance and two points that correspond to the same absolute value but the opposite sign of $\Delta m$. The number of random vectors vectors is fixed to $N_{\eta}=6$ and $N=8$ at (or $N=4$ at half) the maximal reweighting distance. The renormalized quark mass is the average mass of the two quarks after reweighting.

where we replaced $\Delta m$ by the difference of the renormalized masses. Lowest order chiral perturbation theory predicts $q=1$. In order to reveal the mass dependence we plot in the right panel of Fig. $1 \sigma_{1 \mathrm{f}}^{2} /\left(V \Delta \bar{m}^{2} / a^{2}\right)$ and $\left\langle\delta_{1 \mathrm{f}}^{2}\right\rangle \cdot N N_{\eta} /\left(V \Delta \bar{m}^{2} / a^{2}\right)$ as function of the (average) renormalized quark mass after reweighting. The four points for each ensemble correspond to $\pm \max (|\Delta \bar{m}|)$ and half this distance. Note that we neglect errors on the $x$-axis.

There is no visible dependence on the quark mass, so one would conclude $q=q^{\prime}=0$. This result is somewhat puzzling, since for very large mass the fluctuations should go to zero and for very small mass the chiral expansion should describe the data. These results seem to be obtained in an intermediate regime.

\section{Isospin reweighting}
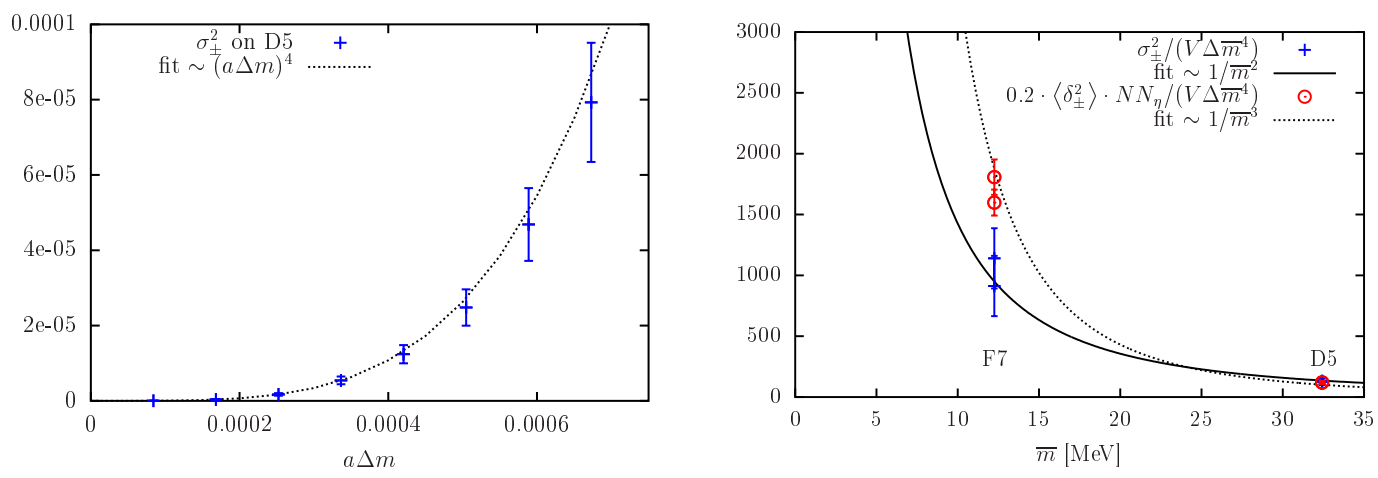

Figure 2: Same as Fig. 1 but for isospin reweighting, i.e., $\sigma_{ \pm}^{2}$ and $\left\langle\delta_{ \pm}^{2}\right\rangle$.

In the case of isospin reweighting the two quarks are reweighted simultaneously in opposite direction, as in Eq. (4.3). The expansions in Eqs. (1.2) and (3.6) work also in this case. We 
demonstrate this in the left panel of Fig. 2 for the ensemble fluctuations. Again the behavior of the stochastic error is similar. Therefore we expect for isospin reweighting

$$
\left\langle\delta_{ \pm}^{2}\right\rangle \approx \frac{k_{\eta, \pm}}{N N_{\eta}} \frac{\Delta \bar{m}^{4} V}{a^{q} \bar{m}^{q}} \quad \text { and } \quad \sigma_{ \pm}^{2} \approx k_{ \pm} \frac{\Delta \bar{m}^{4} V}{a^{q^{\prime} \bar{m}^{q^{\prime}}}},
$$

with $q=3$ indicated by lowest order chiral perturbation theory. In the right panel of Fig. 2 we plot $\sigma_{ \pm}^{2} /\left(V \Delta \bar{m}^{4}\right)$ and $\left\langle\delta_{ \pm}^{2}\right\rangle \cdot N N_{\eta} /\left(V \Delta \bar{m}^{4}\right)$ as function of the renormalized quark mass after reweighting. The two points for each ensemble correspond to $\max (|\Delta \bar{m}|)$ and half this distance. There clearly is a strong dependence on the mass and we also plot the result of one parameter fits of the fluctuations and the stochastic error with $q^{\prime}=2$ and $q=3$. If we assume Eq. (4.5) to be applicable, i.e., higher order terms and finite volume effects to be negligible, the latter fit yields a prediction for the chiral condensate $\Sigma=(325 \mathrm{MeV})^{3}$.

\section{Conclusion}

Reweighting factors in lattice QCD almost always include determinants of ratios of the lattice Dirac operator, i.e., large sparse matrices. The numerical evaluation is necessarily stochastic and based on an integral representation of the determinant. Stochastic estimators with controlled variance can be defined if the determinant is factorized.

Both, the stochastic error of the reweighting factor and its ensemble fluctuations can be expanded in the reweighting distance. For small reweighting distances they are dominated by the first term in this expansion. In the case of one flavor and isospin reweighting the dependence on the renormalized quark mass is analyzed. Whereas for one flavor reweighting there is no dependence in the range considered, for isospin reweighting a strong dependence, $\propto 1 / \bar{m}^{2}$ for the ensemble fluctuations, is found. Although not significant at the quark masses considered here, they will become important at physical quark masses. From the value for F7 in the right panel of Fig. 2, the numbers in Tab. 1 and $\Delta \bar{m}=\left(\bar{m}_{\mathrm{d}}-\bar{m}_{\mathrm{u}}\right) / 2 \approx 1.5 \mathrm{MeV}$ one obtains $\sigma_{ \pm}^{2}(\mathrm{~F} 7) \approx 0.0007$. Compared to F7 physical masses mean a factor four smaller quark mass and a factor $(4 / 3)^{4}$ larger volume to keep finite volume effects small. Thus $\sigma_{ \pm}^{2}$ (phys. mass) $\approx 0.034$, which can have a significant effect on observables.

\section{References}

[1] J. Finkenrath, F. Knechtli, and B. Leder, Nucl. Phys. B877 (2013) 441-456, [arXiv: 1306.3962 ].

[2] A. Hasenfratz, R. Hoffmann, and S. Schaefer, Phys.Rev. D78 (2008) 014515, [arXiv: 0 805.2369].

[3] M. Lüscher and S. Schaefer, Comput.Phys.Commun. 184 (2013) 519-528, [arXiv:1206.2809].

[4] L. Giusti and M. Lüscher, JHEP 0903 (2009) 013, [arXiv: 0812 . 3638].

[5] P. Fritzsch, F. Knechtli, B. Leder, M. Marinkovic, S. Schaefer, et al., Nucl.Phys. B865 (2012) 397-429, [arXiv:1205.5380]. 